\title{
Thermal Diffusion Flow Analysis in Unsaturated Loamy Soil using UPWIND and QUICK Numerical Simulation
}

\author{
Manjit Singh, Mukesh Mann
}

\begin{abstract}
Understanding of energy transport in saturated porous media and unsaturated porous media provides vital information regarding the development of thermal porous reservoirs, industrial filter applications, water reservoirs and low-temperature fluid flow applications. The present paper focus is on presenting the solution of thermal energy diffusion through unsaturated loamy soil using finite difference method approach. The unsteady temperature profile comparison as time function at different spatial locations and as function of spatial location at different time interval are compared using UPWIND and QUICK approach method using thermal diffusion case. MATLAB software is used to simulate above approach for simulation.
\end{abstract}

Keywords: Mathematical modeling, Finite difference method, Loamy soil

\section{INTRODUCTION}

The fluid flow through complex metric of pores through medium (known as porous medium) may contain number of fluids. The applications of above case can be found in many engineering discipline applications such as thermal porous reservoirs, industrial filter applications, water reservoirs and low-temperature fluid flow applications etc. Saturated/ Unsaturated flow problems are derived from Darcy's law [1], analytical works [2], assumption modification ( [3], [4], [5]), validation [6], and further extension of equation [7], [8] based on experiments were carried out by researchers. Enormous publication were published on different modeling concept such as pore-scale model ( [9], and [10]) constant and variable model [11] based on properties relationships like water -clay interaction [12], soil - water characteristic curve [13]. Heat transfer analysis in packed bed using local thermal equilibrium [11], [12] considering and non-local thermal equilibrium (NLTE) condition [14], [15], between phases (fluid / solid) were also published. The mathematical modeling and numerical simulation/ analyses of unsteady flow through unsaturated flow through such case are discussed in detail in [12]. The theoretical and practical aspects are discussed by enormous research paper but research papers based on unsaturated soil numerical simulation are few in quantity.

Revised Manuscript Received on July 01, 2019.

Dr. Manjit Singh, Mechanical Engineering Department, Chandigarh Engineering College, Landran, Mohali, Punjab, India. Email:manjit.cecme@gmail.com

Mukesh Kumar, Mechanical Engineer, Email: mannmukesh2006@gmail.com

\section{LOAMY SOIL ASSUMPTIONS}

The unsaturated loamy soil is considered to obey perfect gas law in the presence of air entrap in soil, it's all hydraulic and thermal physical properties are assumed as homogeneous and isotropic[12].

The dimensionless water saturation is as [11]:

$$
\text { Water saturation, } S_{\mathrm{e}}=\frac{c-c_{\text {res }}}{c_{\text {sat }}-c_{\text {res }}}=\left[\frac{1}{1+(\alpha h)^{n}}\right]^{m} \quad 1
$$

Modified Darcy's law for unsaturated loamy soil based on momentum equation:

$$
u=-K K_{r}(c) \nabla h(c)
$$

A. Mass-balance equation [12]:

$$
\begin{aligned}
\frac{\partial\left(\varepsilon s_{i} \rho_{i}\right)}{\partial t}+\nabla\left(\rho_{i} u_{i}\right) & =0 \quad i=\text { water }, \text { air } \\
\frac{\partial h}{\partial t} & =\frac{\mathrm{K} \frac{\partial}{\partial \mathrm{x}}\left[K_{r} \frac{\partial h}{\partial x}\right]}{\frac{\partial c}{\partial h}}
\end{aligned}
$$

\section{B. Heat transfer model}

1D heat transfer model for loamy soil considering unsaturated flow having constant thermal properties as [12]:

$$
\left(\rho c_{p}\right)_{m} \frac{\partial T}{\partial t}+\left(\rho c_{p}\right)_{f} u \cdot \nabla T=\nabla \cdot\left[\left(k_{m}\right) \cdot \nabla T\right]
$$

\section{Numerical Solution}

Based on first order differencing scheme (backward), the finite difference discretization of above term is:

$$
\begin{aligned}
\frac{h_{i}^{n+1}-h_{i}^{n}}{\Delta t}=\frac{k}{\Delta x^{2}} & {\left[K_{r}^{e} *\left(h_{i+1}^{n+1}-h_{i}^{n+1}\right)\right.} \\
& \left.-K_{r}^{w} *\left(h_{i}^{n+1}-h_{i-1}^{n+1}\right)\right] \\
& *\left(\frac{d c}{d h}\right)^{-1}
\end{aligned}
$$

Similarly, finite difference discretization of heat transfer model considering time domain (implicit) and diffusion (backward differencing) is written as [12]: 


$$
\begin{aligned}
& \frac{T_{i}^{n+1}-T_{i}^{n}}{\Delta t}+\frac{U_{r} T_{r}-U_{l} T_{l}}{\Delta t} \\
& =\frac{\left(k_{m}\right)}{\left(\rho c_{p}\right)_{m}}\left(\frac{T_{i+1}^{n+1}+T_{i-1}^{n+1}-2 T_{i}^{n+1}}{\Delta x^{2}}\right)
\end{aligned}
$$

The velocity driven term $\mathrm{U}$, is numerically solved using second order upwind formulation approach (equation 8 \& 9) and quadratic upstream interpolation for convective Kinematic (QUICK) formulation approach (equation 8,9,10 \& 11) as elaborated [12]:

$$
\begin{gathered}
U_{r}=0.5\left(U_{i}+U_{i+1}\right) \\
U_{l}=0.5\left(U_{i}+U_{i-1}\right) \\
T_{r}=\left(\frac{3}{4}\right) T_{i}+\left(\frac{3}{8}\right) T_{i+1}-\left(\frac{1}{8}\right) T_{i-1} \\
T_{l}=\left(\frac{3}{8}\right) T_{i}+\left(\frac{3}{4}\right) T_{i-1}-\left(\frac{1}{8}\right) T_{i-2}
\end{gathered}
$$

\section{INITIAL AND BOUNDARY CONDITION}

The numerical simulation of thermal diffusion based on initial and boundary condition is carried out considering negative $1 \mathrm{~m}$, matric potential head and bed temperature of at $20{ }^{\circ} \mathrm{C}$ uniformly. The matric potential head at $0 \mathrm{~m}$ (i.e. at 1 Absolute atmospheric conditions) having temperature $50{ }^{\circ} \mathrm{C}$ is allowed to flow. The boundary conditions having a zero derivative towards outflow axial direction follow as:

$\mathrm{h}=1 \mathrm{~m}(-\mathrm{ve})$, at $0 \leq \mathrm{x} \leq \mathrm{L}, \mathrm{t}=0 ; \mathrm{T}=20^{\circ} \mathrm{C}, \mathrm{u}=0$; $\mathrm{h}=0, \quad$ at $\mathrm{x}=0 \quad \mathrm{t}>0 ; \quad \mathrm{T}=50{ }^{\circ} \mathrm{C}$; $\left(\frac{\partial h}{\partial x}\right)_{x=L}=0,\left(\frac{\partial T}{\partial x}\right)_{x=L}=0 ;$ at $\mathrm{x}=\mathrm{L} \quad \mathrm{t}>0$;

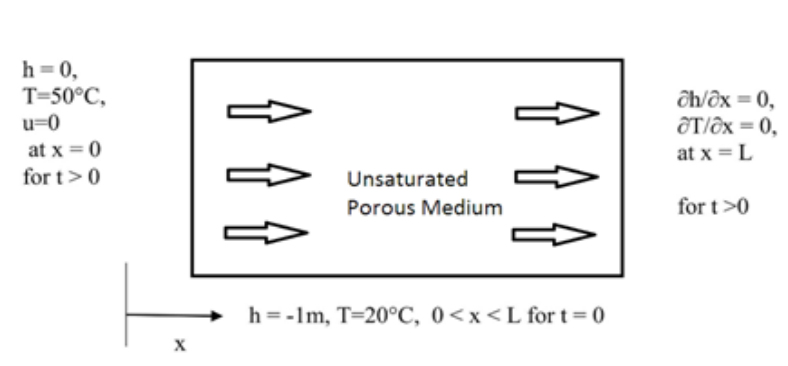

Fig. 1. Schematic representation of loamy soil

The numeric simulation are carried out using on $(\mathrm{R})$ Core (TM) I ${ }^{5}-3230 \mathrm{M}$ CPU processor, WINDOW 8.1 (64-bit) system. Typical computer processing time to solve a single run was about 190 seconds.

\section{VALIDATION OF CODE}

The numerically simulated code results were validated by comparing the experimental work conduct with Singh[16] considering local thermal equilibrium. The temperaturetime profiles at different spatial locations linearly agrees with the simulated results.

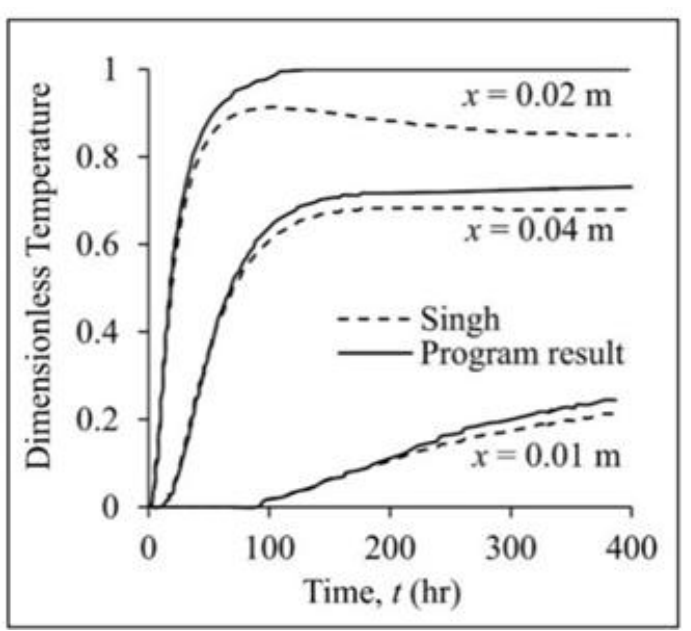

Fig. 2. Results in comparison of Singh[16] with program result at distinguish spatial locations

The loamy sand physical properties as presented in Table 1 are used as input in the program.

Table 1: Loamy sand physical properties

\begin{tabular}{|c|c|c|c|c|}
\hline $\begin{array}{c}\text { Sand } \\
\text { percentage }\end{array}$ & $\begin{array}{c}\text { Clay } \\
\text { percentage }\end{array}$ & Density & $\begin{array}{c}\text { Clay } \\
\text { percentage }\end{array}$ & $\begin{array}{c}\text { Porosity, } \\
\varepsilon\end{array}$ \\
\hline $65 \%$ & $10 \%$ & $\begin{array}{c}2650 \\
\mathrm{Kg} / \mathrm{m}^{3}\end{array}$ & $10 \%$ & 0.453 \\
\hline
\end{tabular}

\section{COMPARISON RESULT BETWEEN SECOND-ORDER UPWIND WITH QUICK APPROACH RESULTS}

The solution obtained from finite difference method solution based on UPWIND with QUICK approach are compared in this section. The comparison of loamy sand soil for unsteady-temperature as a function of time at different axial locations is presented in Fig 3 (a, b, c, and d).

On the basis of transient temperature at specific simulation time using UPWIND and QUICK approach is further presented in Fig. 4 (a, b, c, d, e and f). 

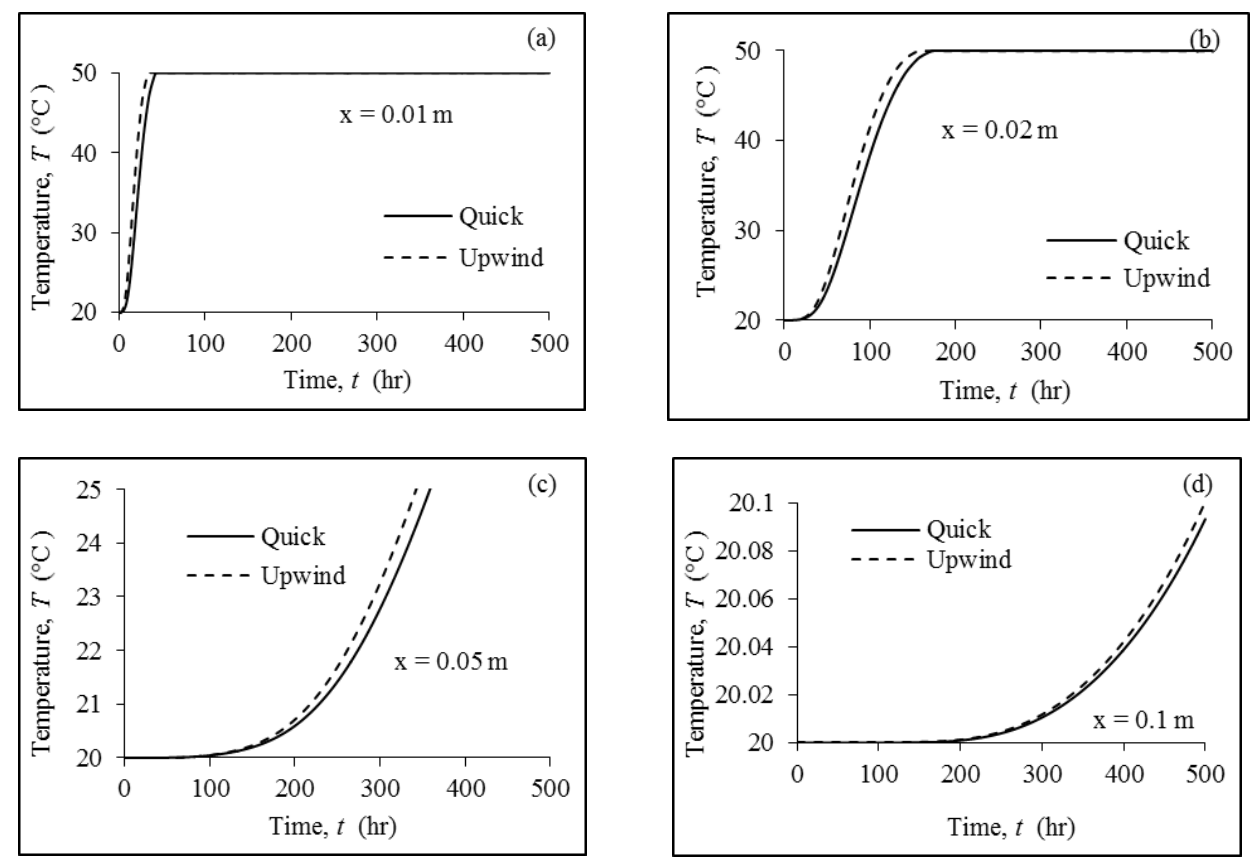

Fig. 3. Comparison of unsteady temperature profile using QUICK and UPWIND method for loamy sand soil
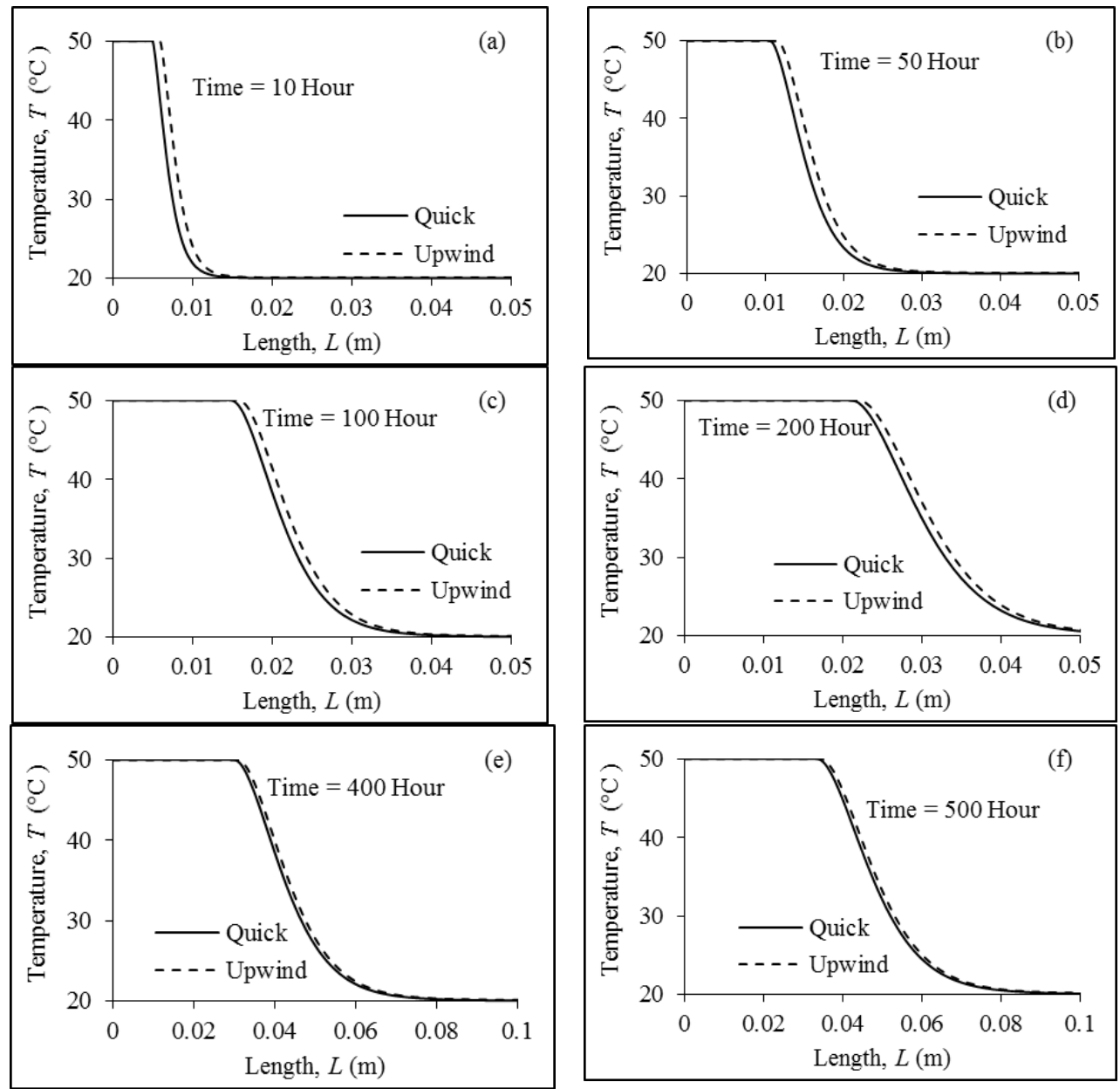

Fig. 4. Comparison of unsteady temperature profile using QUICK and UPWIND method for loamy sand soil 


\section{CONCLUSIONS}

A qualitative validation using QUICK and Upwind approach is carried out. The second order upwind and Quick approach for thermal unsteady temperature response as a function of time at different axial locations for loamy sandalong with temperature profile as a function of spatial locations at different simulation hours such as 10, 50, 100, 200, 400 and 500 hours for loamy sand soil compared and discussed. It is clearly observed that the variation of results is miniature or identical.

\section{REFERENCES}

1. H. Darcy, "Les fontainesPubliques de la Ville de Dijon," Vols. Dalmont, Paris, 1856.

2. M. Muskat, The flow of homogeneous fluid through porous media, United State of America: Mcgraw Hill, 1937.

3. J. Dupuit, "EstudesThéoriques et Pratiques sur le mouvement des Eaux dans les canauxdécouverts et à travers les terrains perméables," (Second ed.)., Paris, Dalmont, 1863.

4. H. Brinkman, "A calculation of viscous force exerted by a flowing fluid on a dense swarm of particles," Applied Scientific Research, vol. 1, pp. 27-34, December 1949.

5. Mukesh Kumar; Manjit Singh; Chanpreet Singh; Ganga Charyulu;, "Modeling for heat conduction transfer through porous media," International Journal of Advance in computing and information technology, Issue-5, pp. 469-474, 2012.

6. W. Stephen, "Flow in Porous Media I: A Theoretical Derivation of Darcy's Law," Transport in Porous Media 1, D. Reidel Publishing Company, pp. 3-25, 1986.

7. S. Irmay, "Sur le mouvement des eaux dans le sol," Rev. Universelle des Mines, vol. 3, no. 4, 1947.

\section{AUTHORS PROFILE}

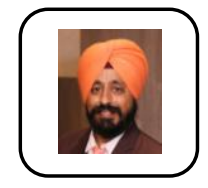

Dr. Manjit Singh is Professor in the Department of Mechanical Engineering at Chandigarh Engineering College, Landran, Mohali, Punjab India. He obtained his M.E and Ph.D. in the area of modeling and simulation (Mechanical System). His research interests are heat transfer and fluid flow, energy exchange, modeling, simulation and computational fluid dynamic. He has published more than 10 research papers and filled 5 patents(Intellectual Property, India).

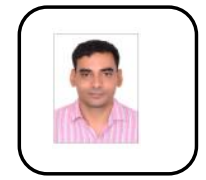

Dr. Mukesh Kumar, Mechanical Engineer has obtained his Ph.D. from Maharishi Dayanand University, Rohtak. His research interest is in industrial engineering, Supply chain management, JIT etc. He has published more than 12 research papers.
8. Manjit Singh; Sandeep Jindal; Amit Gupta; Virender Chahal, "Numerical Analysis of soil-water flow in fixed Horizontal pipe," International journal of theoretical and applied mechanics ISSN 0973-6085, pp. volume 12, number 3, 533-541, 2017.

9. H. K. Dahle, M. A. Celia and S. M. Hassanizadeh, "Bundle-of-Tubes Model for Calculating Dynamic Effects in the CapillaryPressure-Saturation Relationship," Transport in Porous Media, vol. 58, pp. 1-2, 2005.

10. D. Yang, R. P. Currier and Duan Z. Zhang, "Ensemble phase averaged equations for multiphase flows in porous media. Part 1: The bundle-of-tubes model," International Journal of Multiphase Flow, vol. 35, p. 628-639, 2009.

11. Manjit Singh; Chanpreet Singh; D. Gangacharyulu, "Modelling for flow through unsaturated porous media with constant and variable density conditions using local thermal equilibrium," International Journal of Computer Application (0975-8887), pp. 24-30, 2016.

12. Manjit Singh; Chanpreet Singh; D. GangaCharyulu, "Numerical Analysis on variations of thermal and hydraulic properties during water flow through unsaturated soil," in Advances in fluid and thermal engineering, Singapur, Springer, 2019, pp. 357-368.

13. D. G. Fredlund, "Unsaturated Soil Mechanics in Engineering Practice," Journal of geotechnical and geoenvironmental engineering, vol. 132, pp 286-321, 2006.

14. K. Vafai and S. J. Kim, "Analysis of surface enhancement by a porous substrate," ASME J. heat transfer, vol. 112, pp. 700-706, 1990.

15. Y. Mahmoudi and N. Karimi, "Numerical investigation of heat transfer enhancement in a pipe partially filled with a porous material under local thermal non equilibrium condition," International journal of heat and mass transfer, vol. 68, pp. 161-173, 2014.

16. C. Singh, Convective Heat Transfer in Porous Media for Energy Storage, VDM Verlag, 2009.

17. Manjit Singh; Chanpreet Singh; Ganga Charyulu, "Review of hydraulic conductivity and permeability relation in flow through porous medium," International Journal of Engineering Studies, volume 6, pp. 429-434, 2014 . 